\title{
A Constrained Eco-Routing Strategy for Hybrid Electric Vehicles Based on Semi-Analytical Energy Management
}

\author{
Giovanni De Nunzio, Antonio Sciarretta, Ibtihel Ben Gharbia, Luis Leon Ojeda
}

\begin{abstract}
The accuracy of the modern navigation and traffic information systems has increased noticeably. This offers the opportunity of improving the standard powertrain energy management of hybrid electric vehicles (HEVs) by making use of predictive information about the upcoming route. Eco-routing for HEVs aims to optimize fuel consumption by deciding the best route and when to use or recover electrical power based on topological and traffic information. In this work, a simple predictive speed model is used to derive a fast semi-analytical solution of the powertrain energy management. The predicted fuel consumption on each road segment is a function of the desired trade-off between fuel economy and battery use. This modeling complexity is addressed by introducing a novel road network model which takes into account the feasible battery charge variation at road segment level. Also, the routing of HEVs adds several intrinsic constraints and complexity to the classical shortest path problem (SPP). A relaxation of the constrained SPP is proposed to reduce computation time and thus increase user acceptance.
\end{abstract}

Index Terms-Hybrid electric vehicles, energy management, Pontryagin's minimum principle, constrained shortest path.

\section{INTRODUCTION}

Decarbonization and emissions reduction in road transportation are the main drivers for vehicles electrification $(x E V)$. Conservative scenarios of transportation electrification foresee a European market penetration of at least $20 \%$ of xEVs by 2030 [1]. Towards this ambitious achievement, technological developments are mainly focused on increasing both the efficiency/cost ratio of batteries and the travel economy by exploiting data and predictive information about the driving environment. Driving assistance systems tackle the latter aspect by advising the driver on the most energyefficient driving behavior (i.e. route and/or speed trajectory). At route planning level, eco-routing is the strategy that makes use of topological and traffic information about the road network to compute an optimal route in terms of energy consumption. Attractive implementations exist in the literature for standard combustion-engine vehicles (ICEs) [2] and electric vehicles (EVs) [3]. However, eco-routing for HEVs implies two major difficulties in the design both at modeling and routing level.

The first challenge consists in the accuracy of the energy consumption model, whose role is to estimate the energy cost on each elementary road segment. Such an estimation can be performed either by aggregating real-world driving data

G. De Nunzio, IFP Energies nouvelles, Rond-point de l'échangeur de Solaize, BP 3, 69360 Solaize, France, giovanni.de-nunzio@ifpen. fr

A. Sciarretta, I. Ben Gharbia and L. Leon Ojeda, IFP Energies nouvelles, 1 et 4 avenue de Bois-Préau, 92852 Rueil-Malmaison, France, [antonio.sciarretta, ibtihel.ben-gharbia, luis.leon-ojeda] @ifpen.fr
[4], or by using macroscopic map and traffic data to predict driving behavior and feed accurate powertrain models. Model-based approaches have the advantage of being more easily deployable. The energy consumption of HEVs depends on the power split between the two power sources, which is dictated by the energy management strategy (EMS). Optimal EMS [5] aimed at minimizing fuel consumption for a given battery consumption is obtained for a prescribed speed profile using the Pontryagin's minimum principle (PMP), which can be rather time consuming. In an attempt to reduce the computational load of the EMS, approximated optimization methods, such as the selective Hamiltonian minimization (SHM) [6], were introduced. The SHM technique proposes an analytical solution of the optimal control problem (OCP) based on parametric powertrain models and simple rootfinding methods to estimate the co-state value that respects the terminal constraint of the battery state-of-charge (SOC).

The second challenge consists in finding the energyoptimal route while imposing the feasibility of the SOC profile and the terminal SOC constraint. An approach may consist in simply comparing several routing alternatives and choosing the most energy-efficient one [4]. However, for solution optimality, the problem needs to be formulated as a resource-constrained shortest-path problem (RCSPP), which is known to be an NP-hard problem [7][8]. Attempts to solve such a problem for HEVs eco-routing have been made. In [9], a fully-polynomial time approximation scheme, inspired by [10], was proposed to solve the constrained non-polynomial problem. The approximation resides both in the EMS of the vehicle and in the route optimization algorithm. In the vehicle model, simultaneous use of the combustion engine and electric motor (i.e. hybrid mode) is not allowed. Also, the accuracy and the computational effort of the routing solutions is strongly dependent on the approximation parameter and the route length, showing scalability issues. Similarly, in [11] the complexity of the EMS is approximated by simple tradeoff functions between the two power sources, and the routing algorithm is an approximation inspired by [10]. The authors acknowledge that the proposed solution is still impractical in terms of computation time for an end-user deployment of the eco-routing strategy. They propose a workaround by solving a shortest-path problem on a graph expanded with the battery SOC discretization, as a way to satisfy the SOC feasibility constraint by construction. The graph would then be such that only a choice among the feasible options is allowed. However, the accuracy and reliability of this approach are quite dependent on the chosen SOC discretization.

The contributions of this work are threefold. Firstly, a 
semi-analytical solution of the EMS for HEVs is obtained. The procedure is inspired by the SHM, but it can be further simplified and quickened by considering a simple predictive speed model. The speed model predicts the likely accelerations induced by traffic and road infrastructure, and yields piece-wise linear power demand profiles which can be used for the analytical derivation of the solution. The proposed strategy allows for a fast calculation of the fuel consumption per road segment as a function of the desired final SOC. Secondly, a physics-based SOC variation model is introduced. This model allows for an a-priori determination of the feasible final SOC per segment, and is used to introduce a novel SOC-variation augmented graph. Whereas the state-of-the-art proposes graph expansion techniques based on absolute SOC discretization (e.g. from $0 \%$ to $100 \%$ ), the proposed approach locally (i.e. for each segment) expands the graph with a relative discretization within the feasible SOC variation. Lastly, the RCSPP is relaxed and solved via an exact algorithm as a standard SPP, and the SOC constraints are enforced a-posteriori during the iterative search of the desired optimal solution.

The paper is organized as follows. Section II contains the powertrain model, the predictive speed model, and the analytical solution method of the EMS. Section III describes the SOC prediction model for each road segment. The road network model and the relative SOC graph expansion are introduced in Section IV. The constrained routing problem and the proposed solution are described in Section V. Simulations results are in Section VI.

\section{Fuel Consumption Model}

\section{A. Vehicle Powertrain Model}

HEVs have the advantage of combining the benefits of conventional combustion engines and electric motors. For any kind of vehicle, the power request at the wheels for a given speed $v(t)$ is defined as [12]:

$$
P_{w}=m \dot{v}(t) v(t)+\delta_{2} v(t)^{3}+\delta_{1} v(t)^{2}+\delta_{0} v(t)+m g v(t) \sin (\alpha)
$$

where $m$ is the vehicle mass, $g$ is the gravitational acceleration, $\alpha$ is the road slope, and the coefficients $\delta_{i}$ are identified for a considered vehicle. In a parallel HEV, the wheel power (1) results in a power request met by both the combustion engine and the electric motor:

$$
P_{d}=P_{w} \cdot \eta_{t}^{-\operatorname{sign}\left(P_{w}\right)}=P_{e}+P_{m}
$$

where $\eta_{t}$ is the transmission efficiency, $P_{e}$ is the power generated by the combustion engine, and $P_{m}$ is the power generated by the electric motor.

Let us first introduce the combustion engine model. The engine regime is related to the vehicle speed through a transmission ratio $\gamma_{e}(t)$, and is defined as $\omega_{e}(t)=\gamma_{e}(t) v(t) / r$, where $r$ is the wheel radius. Assuming that a discrete transmission (gearbox) is used, $\gamma_{e}$ varies with the gear selected either by the driver (manual transmissions) or by the transmission controller (automatic transmissions). The internal combustion engine fuel consumption can be expressed as:

$$
E_{f}=\int_{0}^{t_{f}} P_{f}(t) \mathrm{d} t
$$

where $t_{f}$ is the duration of the considered time horizon, and $P_{f}$ is the fuel power requested by the engine. This quantity is often modeled by means of engine fuel maps. For online applications, approximated closed-form expressions, such as polynomial models, are used. The Willans-lines approach, for instance, consists in an affine representation relating the available engine power to the fuel power [5]:

$$
P_{f}=a_{n}+b_{n} P_{e}
$$

Note that this function has a discontinuity when the combustion engine is off (i.e. $P_{e}=0$ ), because then $P_{f}=0$.

As for the electric motor, the motor rotational speed is related to the vehicle speed via a fixed transmission ratio $\gamma_{m}$, and is defined as $\omega_{m}(t)=\gamma_{m}(t) v(t) / r$. The electric motor energy consumption is defined as:

$$
E_{b}=\int_{0}^{t_{f}} P_{b}(t) \mathrm{d} t
$$

where $P_{b}$ is the electrochemical power drained from or supplied to the battery. This quantity is usually related to the electric power supplied to or generated by the motor, $P_{m}$, either by motor maps or by approximating polynomial functions, particularly suitable for online use:

$$
P_{b}=c_{n}+d_{n} P_{m}+e_{n} P_{m}^{2}
$$

The coefficients $a_{n}, b_{n}, c_{n}, d_{n}$ and $e_{n}$, with $n \in\left[0, t_{f}\right]$, are generally time-varying and depend on the instantaneous values of the engine rotational speed along the driving profile. In this work, the engine coefficients $a_{n}$ and $b_{n}$ are polynomial functions of the engine regime and are defined as: $a_{n}=8.5 \cdot 10^{-4} \omega_{e}^{2}+5.5 \omega_{e}+22$ and $b_{n}=2.7 \cdot 10^{-5} \omega_{e}+2.4$, with $\omega_{e}$ expressed in RPM. The electric motor coefficients are considered constant: $c_{n}=0, d_{n}=0.95$, and $e_{n}=10^{-6}$.

\section{B. Vehicle Speed Model}

In an eco-routing strategy, the speed profile $v(t)$ on the different road segments is not known a-priori and should be predicted based on topological and traffic information. As presented in a previous work [13], the provided average traffic speed is enriched by taking into account the speed profile disruptions induced by the road signalization and infrastructure. As illustrated in Figure 1, a synthetic speed profile could be generated for every pair of road segments, $\{i-1, i\}$, by defining a transition speed $v_{t, i}$ at the interface between them:

$$
v_{t, i}=\beta \cdot \frac{\bar{v}_{i}+\bar{v}_{i-1}}{2}
$$

where $\bar{v}_{i-1}$ and $\bar{v}_{i}$ are the average traffic speeds provided by the map web services, and $\beta \in[0,1]$ is a parameter depending on the type of interface (e.g. stop sign, traffic light, turning movement, etc.), which could be selected in a deterministic or stochastic fashion. Therefore, the speed variation in the first transient of the profile is modeled as $v_{1}(t)=$ 

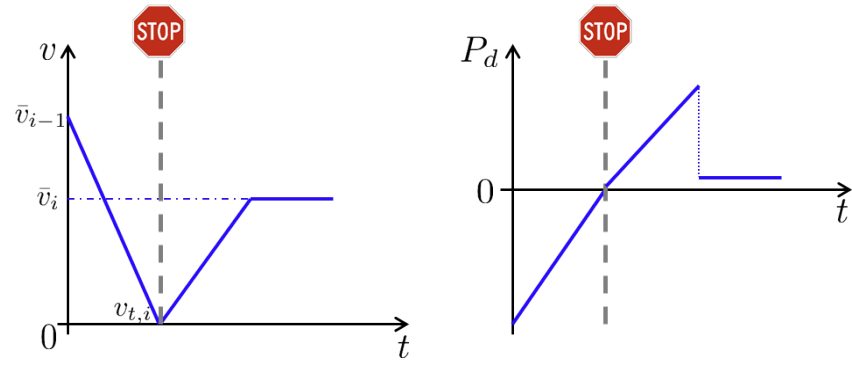

Fig. 1. Example of a synthetic speed profile over two adjacent road segments and the power profile requested from the propulsion system.

$\bar{v}_{i-1}+\operatorname{sign}\left(v_{t, i}-\bar{v}_{i-1}\right) \cdot a \cdot t$, the second transient is modeled as $v_{2}(t)=v_{t, i}+a \cdot t$, while the last portion of the profile, until the end of the considered road segment, is simply the final cruising speed $v_{3}(t)=\bar{v}_{i}$. Thus, the complete speed profile results from the concatenation of the three elementary speed trajectories, as $v(t)=\left[v_{1}(t), v_{2}(t), v_{3}(t)\right]$. Finally, the power demand corresponding to the speed profile, as shown on the right-hand side of Figure 1, is obtained by plugging $v(t)$ in the vehicle model in equations (1)-(2).

\section{Semi-analytical solution of the EMS}

Energy consumption for HEVs is ultimately dictated by the on-board EMS. The goal of the EMS is to minimize the fuel consumption of the vehicle while satisfying state (i.e. speed limits, battery charge sustenance) and input (i.e. minimum and maximum engine and motor torque) constraints. The most important degree of freedom to achieve this goal is represented by the power split, which is defined as the ratio of power delivered by the engine and the motor. Such an OCP is usually formulated by considering the instantaneous cost function as a sum of the fuel consumption and an equivalent fuel consumption related to the battery SOC variation [5]. Within this framework, the Hamiltonian function is:

$$
H\left(P_{m}, v(t)\right)=P_{f}\left(P_{m}, v(t)\right)+s \cdot P_{b}\left(P_{m}, v(t)\right)
$$

The co-state adjoint to the electric power, $s$, is found such that the constraint over the desired final SOC is met. If $s$ is too large, the use of the combustion engine is favored and fuel consumption increases. On the contrary, if $s$ is too small the electric motor use is preferred and the battery SOC decreases. Finally, the optimal power provided by the electric motor is a function of vehicle speed only:

$$
P_{m}^{*}(v(t))=\arg \min _{P_{m}} H\left(P_{m}, v(t)\right)
$$

Let us rewrite the expression of the Hamiltonian function to be minimized, by plugging equations (4) and (6) in (8):

$$
H=a_{n}+b_{n}\left(P_{d}-P_{m}\right)+s \cdot\left(c_{n}+d_{n} P_{m}+e_{n} P_{m}^{2}\right)
$$

Hence, minimization of the Hamiltonian with respect to the electric motor power yields:

$$
P_{m}^{*}=P_{u}=\frac{b_{n}}{2 s e_{n}}-\frac{d_{n}}{2 e_{n}}
$$

As one may notice, the Hamiltonian function is a parametric quadratic function of the electric power $P_{m}$, with parameter $s$. The function has two local minima: one corresponding to the optimal electric motor power in (11), and one corresponding to the operation point when the entire power demand $P_{d}$ is met only by the electric motor (i.e. combustion engine switched off). The latter local minimum is located in a discontinuity, which is generated by the fuel power model in (4) for $P_{e}=0$. These two local minima suggest that the HEV propulsion system is locally optimized either in hybrid mode (i.e. $P_{m}=P_{u}$ and $P_{e}=P_{d}-P_{u}$ ) or in fully-electric mode (i.e. $P_{m}=P_{d}$ ). In particular, when $\left.H\right|_{P_{m}=P_{d}}<\left.H\right|_{P_{m}=P_{u}}$, then the optimal operation is represented by the fully-electric mode. On the contrary, when $\left.H\right|_{P_{m}=P_{d}}>\left.H\right|_{P_{m}=P_{u}}$, then the optimal operation is represented by the hybrid mode. Finally, in the limit case of $\left.H\right|_{P_{m}=P_{d}}=\left.H\right|_{P_{m}=P_{u}}$, the two modes are equally optimal and the EMS can switch between them. Such a limit case and the equality of the two Hamiltonian functions can be explicitly written as follows:

$s\left(c_{n}+d_{n} P_{d}+e_{n} P_{d}^{2}\right)=a_{n}+b_{n}\left(P_{d}-P_{u}\right)+s\left(c_{n}+d_{n} P_{u}+e_{n} P_{u}^{2}\right)$

The desired SOC variation $\Delta=\left(S O C\left(t=t_{f}\right)-S O C(t=\right.$ $0))$ is imposed with the following equation:

$$
\int_{0}^{t_{f}} P_{b} \mathrm{~d} t=-\Delta
$$

The EMS is traditionally solved by using time-consuming offline numerical methods.For every possible value of $s$, a power demand $P_{d}=P_{d, t h r}(s)$, corresponding to the limit case, is obtained from equation (12). This value acts as a threshold for the switch between the two optimal operation modes, and $s$ must be such that equality (13) is satisfied.

In the following, by exploiting the simple form of the synthetic speed profile $v(t)$, a semi-analytical solution method is proposed. Let us assume that the power demand profile $P_{d}$ can be divided and sorted into three distinct elementary power profiles $P_{1}>P_{2}>P_{3}$ corresponding to three different phases, of duration $T_{1}, T_{2}$ and $T_{3}$, respectively. The desired SOC variation $\Delta$ can be imposed by rewriting (13):

$$
\int_{0}^{T_{1}} P_{b 1} \mathrm{~d} t+\int_{0}^{T_{2}} P_{b 2} \mathrm{~d} t+\int_{0}^{T_{3}} P_{b 3} \mathrm{~d} t=-\Delta
$$

where $P_{b 1}, P_{b 2}$ and $P_{b 3}$ are the unknown optimal battery power profiles associated with the three phases $P_{1}, P_{2}$ and $P_{3}$. In the following, for ease of notation, let us denote:

$$
\left.\int_{0}^{T_{i}} P_{b}\right|_{P_{u}} \mathrm{~d} t=E_{b, u, i} \quad \text { and }\left.\quad \int_{0}^{T_{i}} P_{b}\right|_{P_{i}} \mathrm{~d} t=E_{b, i}
$$

with $i \in\{1,2,3\}$ being the index of the considered phase.

As illustrated in Figure 2, depending on the possible location of $P_{d, t h r}$ with respect to the power profiles, seven different cases can be distinguished. In this work, the approach is to enumerate and evaluate the fuel consumption in all of the possible cases. Then the optimal case (i.e. minimum fuel consumption) and the associated $s$ and $P_{u}$ are selected. 


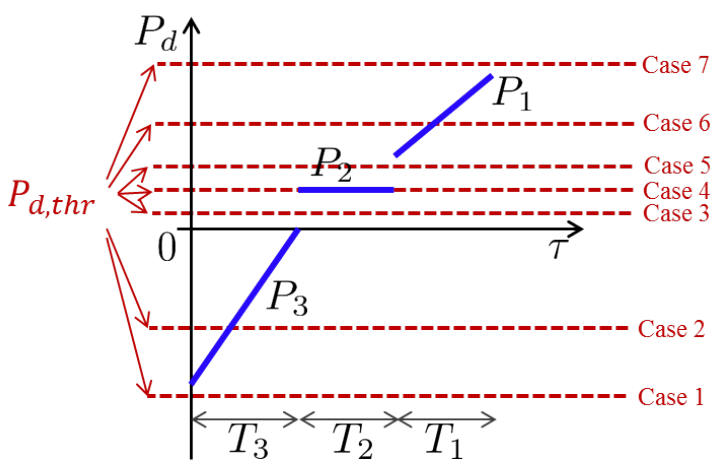

Fig. 2. Schematic of the different cases for the semi-analytical solution of the EMS, depending on the value of $P_{d, t h r}$. The power demand profile $P_{d}$ is divided into distinct power profiles sorted as $P_{1}>P_{2}>P_{3}$.

1) Fully-hybrid mode: In the first case $H\left(P_{d}\right)>H\left(P_{u}\right)$ at every instant, therefore the vehicle remains in hybrid mode over the entire driving profile. The optimal electric motor power $P_{u}$ is such that:

$$
E_{b, u, 3}+E_{b, u, 2}+E_{b, u, 1}+\Delta=0
$$

2) Switch during phase 3: The vehicle is in hybrid mode during phases 1 and 2. The switch from electric mode to hybrid mode takes place during phase 3. $P_{u}$ is calculated from (11) by using the $s$ that satisfies (12). Hence, the energy ratio $\xi$ defining the switch from the fully-electric to the hybrid mode is calculated such that:

$$
\xi E_{b, 3}+(1-\xi) E_{b, u, 3}+E_{b, u, 2}+E_{b, u, 1}+\Delta=0
$$

3) Switch between phases 3 and 2: The vehicle is in hybrid mode during phases 1 and 2, and in fully-electric mode during phase 3 . The optimal electric motor power $P_{u}$ is such that:

$$
E_{b, 3}+E_{b, u, 2}+E_{b, u, 1}+\Delta=0
$$

4) Switch during phase 2: The vehicle is in hybrid mode during phase 1 , and in electric mode during phase 3 . The switch from electric mode to hybrid mode takes place during phase 2. $P_{u}$ is calculated from (11) by using the $s$ that satisfies (12). Hence, the energy ratio $\xi$ defining the switch from fully-electric to hybrid mode is calculated such that:

$$
E_{b, 3}+\xi E_{b, 2}+(1-\xi) E_{b, u, 2}+E_{b, u, 1}+\Delta=0
$$

5) Switch between phases 2 and 1: The vehicle is in hybrid mode during phase 1 , and in fully-electric mode in phases 3 and 2. $P_{u}$ is such that:

$$
E_{b, 3}+E_{b, 2}+E_{b, u, 1}+\Delta=0
$$

6) Switch during phase 1: The vehicle is in electric mode during phases 3 and 2 . The switch from electric mode to hybrid mode takes place during phase 1. $P_{u}$ is calculated from (11) by using the $s$ that satisfies (12). Hence, the energy ratio $\xi$ defining the switch from the fully-electric to the hybrid mode is calculated such that:

$$
E_{b, 3}+E_{b, 2}+\xi E_{b, 1}+(1-\xi) E_{b, u, 1}+\Delta=0
$$

7) Fully-electric mode: In the last case, $H\left(P_{d}\right)<H\left(P_{u}\right)$ at every instant, therefore the vehicle remains in electric mode over the entire driving profile and the optimal solution is ensured by $P_{m}=P_{d}$ :

$$
E_{b, 3}+E_{b, 2}+E_{b, 1}+\Delta=0
$$

Finally, by knowing the optimal electric motor power for each of the above cases, the corresponding fuel consumption of the combustion engine is obtained as:

$$
E_{f}=\int_{0}^{T_{u}} a_{n}+b_{n}\left(P_{d}-P_{u}\right) \mathrm{d} t
$$

where the integration interval $T_{u}$ is equal to the sum of the different time intervals during which the propulsion system is in hybrid mode. The optimal fuel consumption is then the minimum among the consumptions calculated for all the cases. Intuitively, the optimal fuel consumption, for a given power demand $P_{d}$, can be defined as a function of the desired variation of battery charge $\Delta$ (i.e. $E_{f}\left(P_{d}, \Delta\right)$ ).

\section{III. $\triangle$ SOC DISCRETIZATION MODEL}

A specific analysis has been dedicated to the prediction of the achievable SOC variation for a given trip. A training dataset was created by generating 150 trips with randomly chosen parameters: number of road segments, boundary speeds for each segment, average speed, mean road grade, and length of each segment. The OCP minimizing the fuel consumption over each generated trip was solved via the dynamic programming (DP). The goal was to calculate the optimal $\Delta_{i}$ for each road segment $i$ composing the trip, subject to the constraint of invariant total SOC (i.e. $\left|\sum_{i} \Delta_{i}\right| \leq \epsilon$ ). Inspired by the optimal results obtained via the ${ }^{i} \mathrm{DP}$, a deterministic model for the optimal SOC variation per road segment has been proposed. This model states that the optimal SOC variation is a function of the vehicle parameters and the predicted kinetic and potential energy to travel on the road segment. Thus, the optimal $\Delta_{i}$ is given by:

$\Delta_{i}=\rho \frac{0.5 m\left(v_{i, f}^{2}-v_{i, 0}^{2}\right)+m g\left(h_{i, f}-h_{i, 0}\right)}{Q V}=\rho \frac{E_{k i n}+E_{p o t}}{Q V}$

where $Q$ is the battery capacity, $V$ the battery voltage (a total battery energy of $Q V=7.6 \mathrm{kWh}$ was used in this work), $v_{i, f}$ and $v_{i, 0}$ are the speed values at the end and the beginning of the road segment, $h_{i, f}$ and $h_{i, 0}$ are the altitude values at the end and the beginning of the road segment. The correction parameter $\rho$ is tuned in order to minimize the estimation error with respect to the optimal results obtained by the DP. Finally, in order to model the prediction uncertainty and have an estimate of the SOC discretization bounds, the optimal $\Delta_{i}$ 's obtained from the DP were used to compute confidence intervals. In Figure 3, the green circles represent the optimal values of $\Delta_{i}$, and the solid blue line is the proposed optimal SOC variation model. The confidence intervals $\Delta_{i, \min }$ and $\Delta_{i, \max }$ are computed in an intentionally conservative way by defining an envelope of $\pm 1 \%$ around the estimated values. 


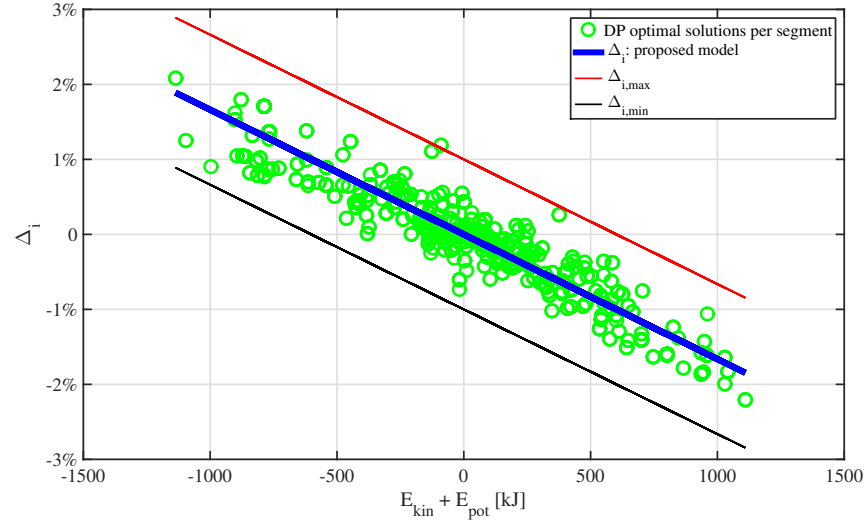

Fig. 3. Validation of the proposed optimal SOC variation model against reference optimal results calculated via the DP. The minimum and maximum bounds correspond to an envelope of $\pm 1 \%$ around the estimated value.

\section{ROAD NETWORK MODEL}

The road transportation network can be modeled as a directed graph. Let $\mathcal{G}=(V, A)$ be such a graph, where $V$ is the set of road intersections (or nodes), and $A$ is the set of road segments $i$ (or links) connecting the nodes of the graph. The vehicle speed model presented in Section II-B depicts interface accelerations between adjacent links, which makes impractical the standard graph representation of the road network for eco-routing applications. In particular, every node of the graph with two or more incoming links is critical because the upstream speed $\bar{v}_{i-1}$ is not unique. Evidently, this prevents from assigning unique energy (or fuel) consumption costs to the links of the graph. Hence, the road network is modeled as a directed line graph, which can be thought of as the graph of the allowed maneuvers and is defined as follows [13]:

Definition 1: The line graph $\mathcal{L}(\mathcal{G})=\left(A, A^{*}\right)$ of a directed graph $\mathcal{G}=(V, A)$ has a node for each link in $\mathcal{G}$ and each link represents a pair of adjacent links in $\mathcal{G}$.

Therefore, a weighting function for the links of the line graph $w^{*}: A^{*} \rightarrow W^{*}$, where each weight represents the optimal fuel consumption to perform the corresponding maneuver $k \in A^{*}$, is defined as:

$$
W_{f, k}^{*}=E_{f, k}\left(P_{d, k}, \Delta\right)
$$

where $P_{d, k}$ is the predicted power demand over link $k \in A^{*}$.

\section{A. $\triangle$ SOC-Augmented Graph}

The graph weights in (25) can take on infinitely-many values depending on the desired battery consumption over the graph links. The problem of finding the optimal $\Delta$ for each link of the graph while searching the optimal route from an origin to a destination has been addressed before in the literature [11]. However, the proposed solution strategies are often impractical due to large computational cost. In this work, the line graph $\mathcal{L}(\mathcal{G})=\left(A, A^{*}\right)$ is augmented by creating as many copies of each link of the graph as the number of pre-defined possible values of SOC variation $\Delta$. Thus, let us define a $\Delta$ SOC-augmented graph $\mathcal{L}_{\Delta}(\mathcal{G})=$
$\left(A, A_{\Delta}^{*}\right)$ and a new weighting function for the links of the augmented graph $w_{\Delta}^{*}: A_{\Delta}^{*} \rightarrow W_{\Delta}^{*}$. Each weight represents the optimal fuel consumption to perform the corresponding maneuver $k_{j} \in A_{\Delta}^{*}$ for a given $\Delta_{j}$, and is defined as:

$$
W_{f, k_{j}, \Delta}^{*}=\left.E_{f, k}\left(P_{d, k}\right)\right|_{\Delta_{k, j}}
$$

with $\Delta_{k, j} \in\left[\Delta_{k, \min }, \Delta_{k, \max }\right]$. All the copies $k_{j} \in A_{\Delta}^{*}$ corresponding to the maneuver $k \in A^{*}$ share the same power demand profile $P_{d, k}$. The copies differ from one another in terms of fuel consumption because of the associated $\Delta_{k, j}$. The number $j$ of copies, or SOC variation levels, is a design parameter, in a trade-off between discretization accuracy and computational burden. Furthermore, the variation range of $\Delta_{k, j}$ depends on the physical properties of the maneuver $k \in A^{*}$. Note that, the $\triangle \mathrm{SOC}$ discretization model and confidence intervals presented in Section III also hold for the maneuvers $k \in A^{*}$, and can be used to decide the most representative and meaningful discretization $\Delta_{k, j} \in$ $\left[\Delta_{k, \text { min }}, \Delta_{k, \text { max }}\right]$ in the $\Delta$ SOC-augmented graph. Finally, it is important to observe that the proposed graph expansion offers a much higher precision as compared to other approaches, in which the SOC is discretized in absolute between a minimum and a maximum charge level [11][14].

\section{Routing Problem}

For an HEV, eco-routing is defined as the problem of finding the route that minimizes fuel consumption, while respecting battery charge constraints. Some of these constraints aim to respect the physical bounds of the energy storage, in order not to excessively deplete or charge the battery. Some other constraints depend on the vehicle powertrain architecture, and are typically associated with a desired final level of SOC at the end of the trip.

\section{A. Problem Formulation}

In this work, the eco-routing problem is solved on the $\Delta$ SOC-augmented graph $\mathcal{L}_{\Delta}(\mathcal{G})=\left(A, A_{\Delta}^{*}\right)$, and may be formulated as follows:

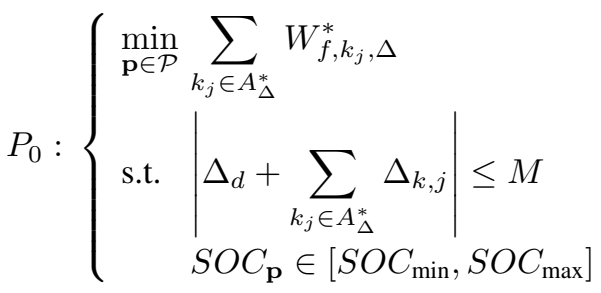

where $\mathbf{p}$ is the path in the set of all paths $\mathcal{P}$ that solves the routing problem. The final SOC is constrained to differ of at most $M$ from a desired value of battery charge by (27b). The desired SOC variation $\Delta_{d}$ is defined as the difference between the desired final SOC and the initial one. Finally, the SOC profile of the optimal path $\mathbf{p}$ must be feasible, in compliance with (27c). Such an optimization problem is known in the literature as resource-constrained SPP (RCSPP). The presence of resource constraints makes the RCSPP an NPhard problem, even if the graph is acyclic and both costs and resources are positive [8]. In this framework, the road transportation graph is cyclic by nature, the costs $W_{f, k_{j}, \Delta}^{*}$ 
(i.e. fuel consumption) are positive, and the resources $\Delta_{k, j}$ (i.e. relative $\mathrm{SOC}$ variation) can be negative.

In order to reduce the complexity, a more tractable problem formulation can be introduced as follows:

$$
P_{1}: \begin{cases}\min _{\mathbf{p} \in \mathcal{P}} & \sum_{k_{j} \in A_{\Delta}^{*}} \lambda C_{f, k_{j}, \Delta}-(1-\lambda) R_{k, j} \\ \text { s.t. } & S O C_{\mathbf{p}} \in\left[S O C_{\min }, S O C_{\max }\right]\end{cases}
$$

where the two terms of the objective function are defined as:

$$
C_{f, k_{j}, \Delta}=\frac{W_{f, k_{j}, \Delta}^{*}}{W_{f, \max }^{*}} \quad \text { and } \quad R_{k, j}=\frac{\Delta_{k, j}}{\Delta_{\max }}
$$

with $W_{f, \max }^{*}$ and $\Delta_{\max }$ being the normalization factors for the fuel consumption and the SOC variation, respectively. The problem $P_{1}$ is still an RCSPP, but the constraint on the desired final SOC has been transformed into an additional term of the objective function. In particular, problem $P_{1}$ aims to minimize fuel consumption while maximizing battery energy recovery. Such a formulation yields a bi-objective optimization problem, cast as a single-objective via weightedsum scalarization. The optimization weight $\lambda \in[0,1]$ defines the trade-off between the two objectives, and should be sought such that the final SOC for the optimal path $\mathbf{p}$ is within the desired range, according to (27b).

Note that, although HEVs in reality could use cycles to recharge the battery, in this work the optimal path $\mathbf{p}$ is assumed to be simple, therefore acyclic. Turning in circles to recharge the battery would penalize travel time, which would have a negative impact on the driver's acceptance. As a consequence, problem $P_{1}$ could be solved by using the constrained Bellman-Ford algorithm [15] (graph weights could be negative), which keeps track of the partial paths and discards the unacceptable ones. However, the constrained Bellman-Ford algorithm is still an impractical solution because its time complexity grows exponentially with the graph size. Therefore, let us propose a final unconstrained formulation:

$$
P_{2}: \min _{\mathbf{p} \in \mathcal{P}} \sum_{k_{j} \in A_{\Delta}^{*}} \lambda C_{f, k_{j}, \Delta}-(1-\lambda) R_{k, j}
$$

Constraints (27b) and (27c) are verified a-posteriori on the optimal paths during the process of seeking $\lambda$.

\section{B. Solution Approach}

Given a pair of origin and destination nodes $\left(i_{o}, i_{d}\right) \in$ $A$, as well as an initial level of battery charge $S O C(t=$ $0)=S O C_{0}$, the objective of the solution approach is to find the parameter $\lambda \in[0,1]$ such that the SOC constraints are satisfied. Problem $P_{2}$ is an SPP formulated as a bi-objective optimization in the weighted-sum paradigm.

The proposed solution strategy, as illustrated in Algorithm 1 , is based on an initial iterative search of $\lambda$ by means of a search parameter $\lambda_{\text {step }}$. The value of this parameter is variable and adaptive depending on the solution sensitivity. The search is iterated as long as $\lambda$ yields an optimal path with a total $\Delta$ SOC below the desired range, as in (27b). Note that, making a standard initialization choice of $\lambda=0$ would

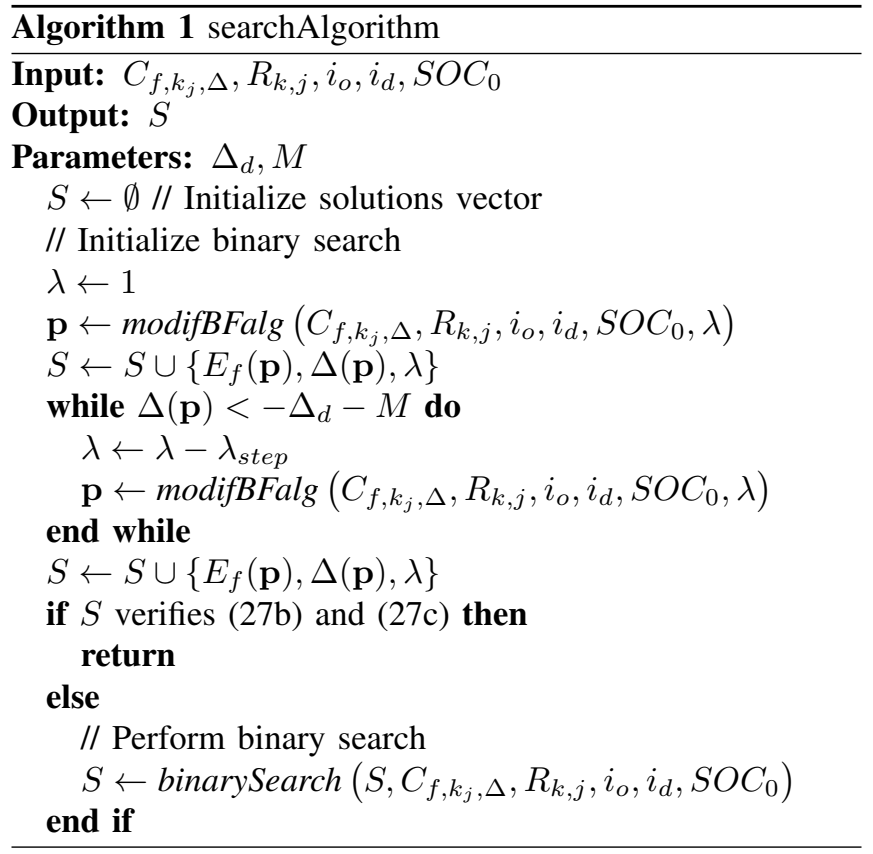

result in solving a longest-path problem (NP-hard), which is both intractable and uninteresting for our needs. At this point, the solutions stored in $S$ are tested for verification of the constraints (27b) and (27c). At the end of the iterative search, two extreme values of $\lambda$ and two feasible paths are available in $S$. Therefore, if neither of the solutions in $S$ verifies the final SOC constraint in (27b), a binary search is started until such a solution is found. The binarySearch used in Algorithm 1 was inspired by the one used in [13], but modified to include the verification of the SOC constraints and the termination condition to halt the recursion when a feasible solution is found. The modifBFalg is a slightly modified version of the Bellman-Ford algorithm that was already optimized and used in [13]. The modification consists in updating not only the fuel cost during the standard relaxation phase, but also the final SOC value to keep track of the SOC profile associated with the optimal route.

\section{RESUlts}

The simulation experiments were conducted on a chargesustaining HEV (i.e. $\Delta_{d}=0$ and $M=5$ ), which makes the problem of seeking $\lambda$ more challenging. In fact, for a chargedepleting HEV a trivial choice of $\lambda=1$ might be suitable for most cases. The routing graph represents the road network of the city center of Paris, and the associated $\triangle$ SOC-augmented graph has $\left|A_{\Delta}^{*}\right|=403079$ links. The traffic information was provided by HERE Maps for the day of April 3rd, 2018, at 06:00 UTC (corresponding to a peak hour). The objective of the simulation experiments is twofold: showing the ecorouting results in terms of fuel consumption and SOC profile over the suggested route, and providing a statistical analysis of the computation time.

For a random selection of an origin/destination (O/D) pair, the suggested eco-route is shown in Figure 4, while the fuel consumption and SOC profile, as well as the 


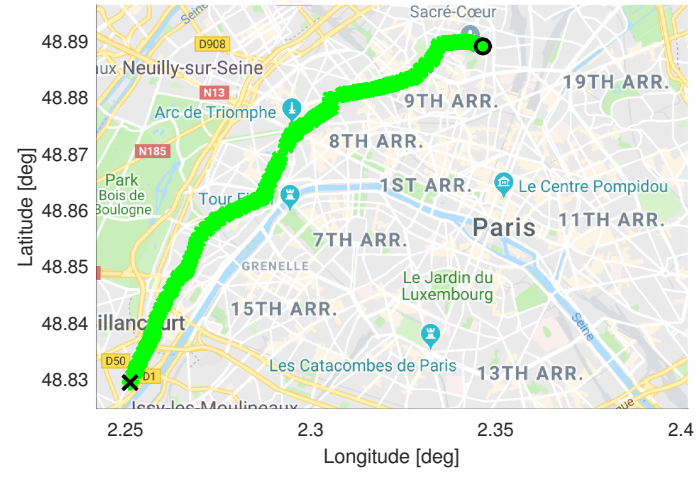

Fig. 4. Map display of the calculated eco-route.

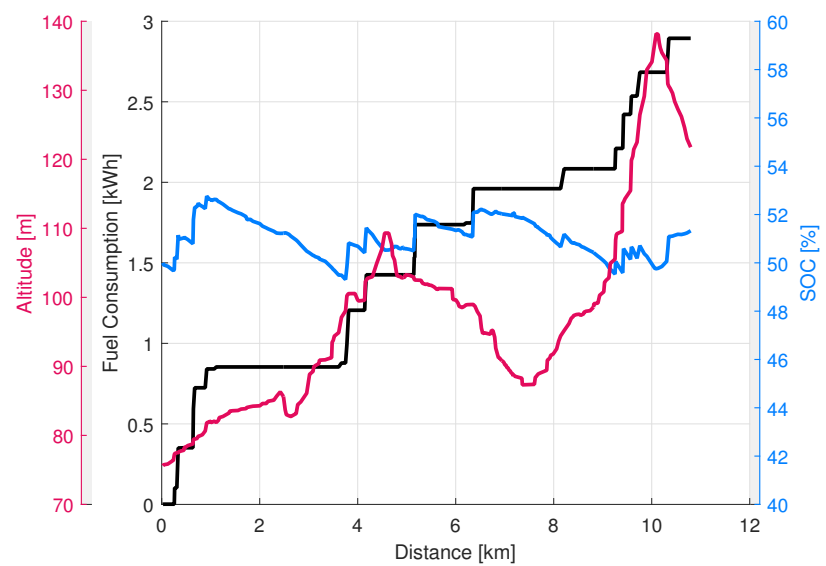

Fig. 5. Fuel consumption, battery state-of-charge and altitude profiles as a function of the traveled distance along the calculated eco-route.

route altitude profile, are shown in Figure 5. The optimal route and energy management yield a fuel consumption of $2.89 \mathrm{kWh}$ (i.e. about $3 \mathrm{~L} / 100 \mathrm{~km}$ ) with an associated SOC profile starting at $50 \%$ and ending at $51.6 \%$, in compliance with the problem constraints. This optimal solution was obtained for a selection of $\lambda=0.8$. It is important to mention that the single-objective problem of minimizing only fuel consumption for $\lambda=1$ yields a different optimal route and, evidently, a different optimal energy management. In particular, given the large battery capacity, the optimal route in that case would trivially correspond to a fully-electric mode with a final SOC of $34.7 \%$, thus violating the final SOC constraint. The proposed strategy was implemented in MATLAB on a computer with CPU Intel i7-4810MQ at $2.8 \mathrm{GHz}$ and $16 \mathrm{~GB}$ of RAM. In terms of computation time, for a random selection of one hundred O/D pairs within the routing graph, the average computation time was 56 seconds, with a standard deviation of 33 seconds.

\section{CONCLUSIONS}

This work addresses the problem of eco-routing for HEVs and proposes a practical implementation which aims to increase user acceptance by combining solution accuracy and low computation time. The proposed semi-analytical EMS allows for fast and accurate solutions, comparable to the optimal ones calculated by the PMP. The $\triangle \mathrm{SOC}$ becomes a model parameter which can be set to determine the optimal power split to achieve the desired final battery charge. Such a parameter can be set in a realistic way by means of a $\triangle$ SOC prediction model, based on the physics of the problem. The feasible SOC variation thus obtained is used to define a $\triangle \mathrm{SOC}$ graph expansion for the routing problem. Such a technique allows for a much more accurate SOC variation because the discretization can be very fine but only locally and in terms of relative variation. The optimal routing problem is relaxed from an RCSPP to a standard SPP by means of an iterative search of the optimal path. The optimization constraints, dictated by the HEVs powertrain characteristics, are enforced a-posteriori for route feasibility.

\section{ACKNOWLEDGMENTS}

This project has received funding from the European Union's Horizon 2020 research and innovation program under grant agreement No. 724095 - ADVICE.

\section{REFERENCES}

[1] ERTRAC, "European Roadmap Electrification of Road Transport," Tech. Rep., 2017.

[2] Y. M. Nie and Q. Li, "An Eco-Routing Model Considering Microscopic Vehicle Operating Conditions," Transportation Research Part $B$, vol. 55, pp. 154-170, 2013.

[3] S. Kluge, C. Santa, S. Dangl, S. Wild, M. Brokate, K. Reif, and F. Busch, "On the Computation of the Energy-Optimal Route Dependent on the Traffic Load in Ingolstadt," Transportation Research Part C, vol. 36, pp. 97-115, 2013.

[4] L. Zhu, J. Holden, and J. Gonder, "Navigation API Route Fuel Saving Opportunity Assessment on Large-Scale Real-World Travel Data for Conventional Vehicles and Hybrid Electric Vehicles," in Transportation Research Board 97th Annual Meeting, 2018.

[5] L. Guzzella and A. Sciarretta, Vehicle Propulsion Systems. SpringerVerlag Berlin Heidelberg, 2013.

[6] J. Zhao and A. Sciarretta, "Design and Control Co-Optimization for Hybrid Powertrains: Development of Dedicated Optimal Energy Management Strategy," IFAC-PapersOnLine, vol. 49, no. 11, pp. 277284, 2016.

[7] J. E. Beasley and N. Christofides, "An Algorithm for the Resource Constrained Shortest Path Problem,' Networks, vol. 19, no. 4, pp. 379 394, 1989.

[8] L. Di Puglia Pugliese and F. Guerriero, "A Survey of Resource Constrained Shortest Path Problems : Exact Solution Approaches,' Networks, vol. 62, no. 3, pp. 183-200, 2013.

[9] M. M. Nejad, L. Mashayekhy, and D. Grosu, "Optimal Routing for Plug-in Hybrid Electric Vehicles,” Transportation Science, vol. 51, no. 4, pp. 1304-1325, 2017.

[10] R. Hassin, "Approximation schemes for the restricted shortest path problem," Mathematics of Operations Research, vol. 17, no. 1, pp. 36-42, 1992.

[11] M. Strehler, S. Merting, and C. Schwan, "Energy-efficient shortest routes for electric and hybrid vehicles," Transportation Research Part $B$, vol. 103, pp. 111-135, 2017.

[12] A. Sciarretta, G. De Nunzio, and L. L. Ojeda, "Optimal Ecodriving Control: Energy-Efficient Driving of Road Vehicles as an Optimal Control Problem," IEEE Control Systems Magazine, vol. 35, no. 5, pp. 71-90, 2015.

[13] G. De Nunzio, L. Thibault, and A. Sciarretta, "Bi-Objective EcoRouting in Large Urban Road Networks," in IEEE 20h International Conference on Intelligent Transportation Systems, 2017, pp. 57-63.

[14] M. W. Fontana, "Optimal Routes for Electric Vehicles Facing Uncertainty, Congestion, and Energy Constraints," Ph.D. dissertation, Massachusetts Institute of Technology, 2013.

[15] R. Widyono, "The Design and Evaluation of Routing Algorithms for Real-time Channels," Tenet Group, Tech. Rep., 1994. 